\title{
Structure and evolution of star-forming gas in late-type spiral galaxies
}

\section{Kambiz Fathi ${ }^{1,2}$, John E. Beckman ${ }^{1,3}$, Almudena Zurita ${ }^{4}$, Mónica Relaño $^{4}$, Johan H. Knapen ${ }^{1,5}$, Göran Östlin ${ }^{2}$, Claude Carignan ${ }^{6}$, Laurent Chemin $^{7}$, Olivier Daigle ${ }^{6}$ and Olivier Hernandez ${ }^{6}$}

${ }^{1}$ Instituto de Astrofísica de Canarias, C/ Vía Láctea s/n, 38200 La Laguna, Tenerife, Spain email: fathi@iac.es

${ }^{2}$ Stockholm Observatory, AlbaNova University Center, 10691 Stockholm, Sweden

${ }^{3}$ Consejo Superior de Investigaciones Científicas (SCIC), Spain

${ }^{4}$ Depto. de Física Teórica y del Cosmos, Universidad de Granada, 18071 Granada, Spain

${ }^{5}$ Centre for Astrophysics Research, University of Hertfordshire, Hatfield, Herts AL10 9AB, UK

${ }^{6}$ Université de Montréal, C.P. 6128 succ. centre ville, Montréal, QC, Canada H3C 3J7

${ }^{7}$ Observatoire de Paris, Section Meudon, GEPI, CNRS-UMR 8111, Meudon, France

\begin{abstract}
We study two dimensional Fabry-Perot interferometric observations of the nearby face-on late-type spiral galaxy, NGC 628. We investigate the role of the individual H II regions together with the large-scale gravitational mechanisms which govern star formation and overall evolution in spiral galaxies. Our kinematical analysis (reinforced by literature maps in HI and $\mathrm{CO}$ at lower angular resolution) enables us to verify the presence of an inner rapidly rotating inner disk-like component which we attribute to long term secular evolution of the large-scale spiral arms and oval structure. We find that gas is falling in from the outer parts towards the bluer central regions. This could be an early phase in the formation of a pseudo-bulge. We find signatures of radial motions caused by an $m=2$ perturbation, which are likely to be responsible for the inflow of material forming the circumnuclear ring and the rapidly rotating inner structure.
\end{abstract}

Keywords. galaxies: evolution, galaxies: structure, galaxies: kinematics and dynamics, galaxies: ISM, galaxies: individual (NGC 628)

\section{Introduction}

The line-of-sight velocity and velocity dispersion $(\sigma)$ are important parameters in determining the flattening of the various disc layers, the intrinsic shape of the dark matter component, and the nature and extent of disc-halo interactions. If the gaseous layers in the disc are isothermal, the atomic and molecular gas should have similar scale-heights. However, due to the collective effects of gravitational instabilities, dissipation, and feedback from star-forming regions, the atomic gas has been observed to have a distribution different from that of its molecular counterparts.

\section{Our Programme}

We have launched an extensive effort to analyse the processes that govern the ionized gas and compare them with those that control the neutral gaseous components in spiral galaxies (see Fathi et al. 2007). We use Fabry-Perot interferometry with instrumentation presented in Hernandez et al. (2003) and scan the $\mathrm{H} \alpha$ emission-line. Our observations yield the distribution and kinematics of the $\mathrm{H} \alpha$-emitting gas. Quantifying the kinematic 

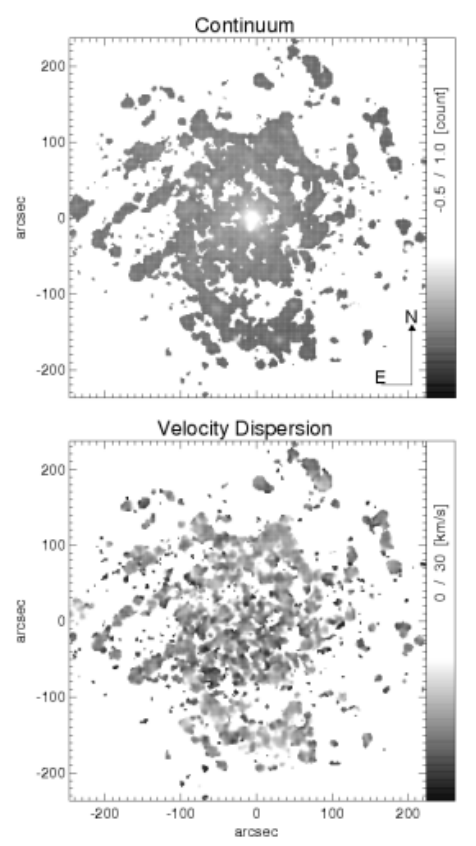
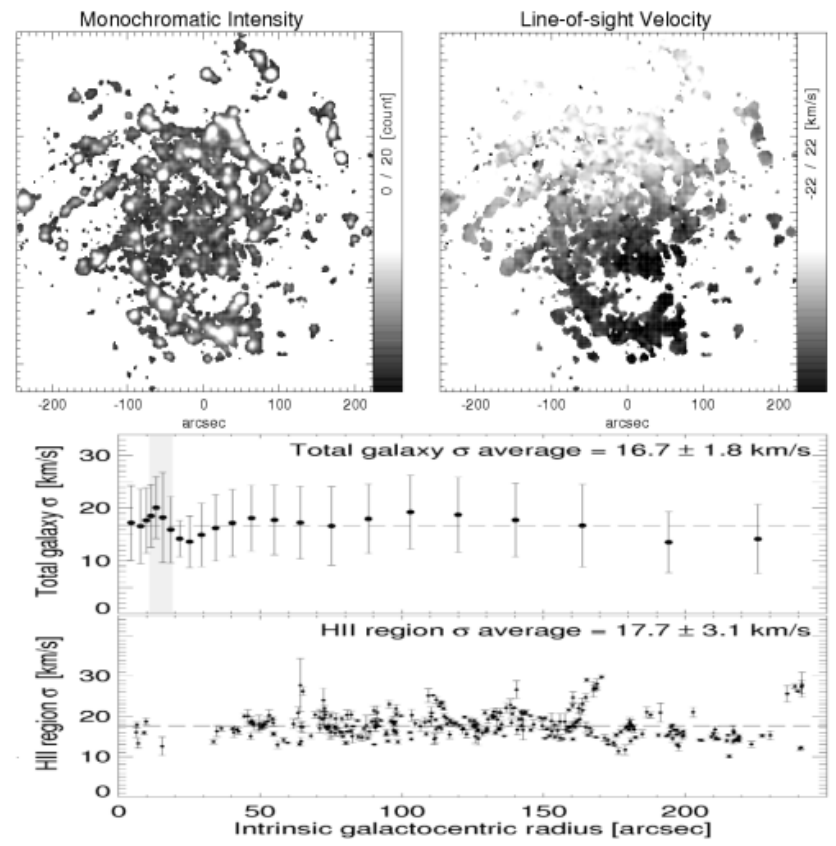

Figure 1. The distribution and kinematics of the H $\alpha$ emitting gas in NGC 628 (top), and the velocity dispersion map with its galactocentric profile (bottom). The velocity field has been quantified using the harmonic decomposition technique, and the $\sigma$ of the individual $\mathrm{H}$ II regions has been derived after using our H II region catalogue to identify them. (see Fathi et al. 2007).

parameters, and comparing with those of the neutral gas will constrain the dynamics of the star-forming regions.

We have chosen NGC 628 for a pilot study in which we demonstrate the power of our state-of-the-art methods to study the properties of the ionized gas (mainly H II regions but also diffuse ionized gas) and their relation with the global kinematic parameters (e.g., Zurita et al. 2004, Fathi et al. 2005). A key feature of the velocity field of NGC 628 is its regularity. The global effects of any asymmetries, such as the oval distortion, are small, and in any case not very easy to detect in a face-on system. However, our detailed kinematic analysis has revealed the presence of a disc-like component in the inner kpc around the nucleus. The $\sigma$ map shows widely distributed star-formation in the disc plane, and that the emission from unresolved $\mathrm{H}$ II regions probably dominates any emission from the diffuse component. The radial $\sigma$-profile shows a nearly constant value of $\approx 17 \mathrm{~km} / \mathrm{s}$ (more than twice that for the $\mathrm{CO}$ and $\mathrm{H}$ I) out to 12 kiloparsec. Our findings for NGC 628 are presented in Fig. 1, and summarized in the abstract of this brief article.

\section{Acknowledgements}

We thank the secretarial staff of the IAC, the LOC, and the SOC for an enjoyable and stimulating conference.

\section{References}

Fathi, K., van de Ven, G., Peletier, R.F. et al. 2005, MNRAS , 364, 773

Fathi, K., Beckman, J.E., Zurita, A. et al. 2007, A $\mathscr{E} A$, submitted

Hernandez, O., Gach, J-L., Carignan, C., \& Boulesteix, J. 2003, SPIE, 4841, 1472

Zurita, A., Relaño, M., Beckman, J.E., \& Knapen, J.H. 2004, A\&A, 413, 73 